\title{
Prognostic significance of myocardial fibrosis in hypertrophic
} cardiomyopathy using cardiovascular magnetic resonance Rory O'Hanlon*1, Agata Grasso', Michael Roughton², James C Moon ${ }^{3}$, Susan Clarke ${ }^{1}$, Ricardo Wage ${ }^{1}$, Jess Webb ${ }^{1}$, Meghana Kulkarni ${ }^{1}$, Dana Dawson ${ }^{1}$, Leena Sulaibeekh ${ }^{1}$, Bud Chandrasekaran ${ }^{1}$, Chiara BucciarelliDucci $^{1}$, Ferdinando Pasquale ${ }^{3}$, Martin R Cowie ${ }^{4}$, Wiliam J McKenna ${ }^{3}$, Margaret Burke ${ }^{5}$, Mary Sheppard ${ }^{1}$, Perry M Elliot ${ }^{3}$, Dudley J Pennell ${ }^{1}$ and Sanjay K Prasad ${ }^{1}$

Address: ${ }^{1}$ Royal Brompton Hospital, London, UK, ${ }^{2}$ R-Squared Statistics, London, UK, ${ }^{3}$ The Heart Hospital, London, UK, ${ }^{4}$ Imperial College, London, UK and ${ }^{5}$ Harefield Hospital, London, UK

* Corresponding author

from I3th Annual SCMR Scientific Sessions

Phoenix, AZ, USA. $21-24$ January 2010

Published: 21 January 2010

Journal of Cardiovascular Magnetic Resonance 20 I0, I2(Suppl I):O50 doi:I0.1 I86/I532-429X-I2-SI-O50

This abstract is available from: http://jcmr-online.com/content/I2/SI/O50

(c) 2010 O'Hanlon et al; licensee BioMed Central Ltd.

\section{Introduction}

The role of myocardial fibrosis in the prediction of sudden death and heart failure in hypertrophic cardiomyopathy (HCM) is unclear.

\section{Purpose}

We sought to investigate the prognostic significance of fibrosis detection by cardiovascular magnetic resonance (CMR) to predict major clinical events in HCM using the late gadolinium-enhanced (LGE) technique.

\section{Methods}

A prospective cohort study of 217 consecutive HCM patients followed for $3.1+1.7$ years to determine the role of fibrosis detected using LGE-CMR on morbidity and mortality.

\section{Results}

LGE was present in 136/217 (LGE+, 63\%). Thirty four of the 136 patients (25\%) in the LGE+ group and 6/81 $(7.4 \%)$ in the LGE- group reached the combined primary endpoint of cardiovascular death, unplanned cardiovascular admission, sustained VT/VF, or appropriate ICD dis- charge, (HR 3.4, p = 0.006). In the LGE+ group, overall risk increased with the percentage of LGE present (HR 1.03 per percent LGE increase, $\mathrm{p}=0.008$ ). The risk of unplanned heart failure admissions, deterioration to NYHA III or IV, or heart failure related death was greater in LGE+ group (HR 2.5, p = 0.021), and this risk increased as the percentage of LGE increased (HR 1.03 per percent LGE increase, $p=0.017)$. All relationships remained significant after multivariate analysis. The overall percentage of LGE was an important univariate predictor for arrhythmic endpoints (sustained VT/VF, appropriate ICD discharge, SCD), HR 1.05 per percent LGE increase, $\mathrm{p}=$ $0.014)$, but did not reach significance after multivariate analysis.

\section{Conclusion}

In patients with HCM, myocardial fibrosis is an independent predictor of adverse outcome particularly due to heart failure 